\title{
Progress and Prospects: Gene Therapy Clinical Trials (Part 2)
}

This is the second part of a review summarizing progress and prospects in gene therapy clinical research. Twenty key diseases/strategies are succinctly described and commented on by leaders in the field. This part includes clinical trials for skin diseases, neurological disorders, HIVIAIDS, ornithine transcarbamylase deficiency, $\alpha_{1}$-antitrypsin deficiency, haemophilia and cancer.

Eric Alton, Stefano Ferrari and Uta Griesenbach

(Progress and Prospects Series Editors)

Gene Therapy (2007) 14, 1555-1563; doi:10.1038/sj.gt.3303033

Keywords: clinical trials; skin diseases; Huntington's disease; Alzheimer's disease; HIVIAIDS; OCT; hemophilia; ADA-SCID; cancer

\section{In brief}

\section{Progress}

- Gene therapy clinical trials for cancer are moving rapidly from phase I to III and the first anti-cancer gene therapy drug has been licensed in China.

- Some serious adverse events following gene therapy have occurred, but progress has been made in understanding and overcoming these problems.

- Clinical trials involving genetic modification of stem cells other than bone-marrow derived (for example skin precursor cells) have been carried out.

- Ex vivo transduction of stem cells with integrating retroviruses ensures high and persistent level of gene transfer and is a promising strategy for several diseases.

\section{Prospects}

- Lentiviral vectors will move from preclinical safety assessment studies to clinical trials.

- Studies to evaluate the genotoxicity of any gene transfer vector will become a fundamental feature of gene therapy research.

- Stem cells from a range of different tissues/organs will become the target cells for many gene therapy applications.

\section{Skin diseases}

\section{G Meneguzzi}

Inserm, University of Nice-Sophia Antipolis, Nice, France

For a number of severe and untreatable diseases affecting the skin, the establishment of the phenotype/genotype relationship has made the development of therapeutic approaches based on cutaneous gene transfer attractive. Indeed, the skin is accessible to direct in vivo gene targeting and, with respect to ex vivo gene transfer, there is the capacity to reconstruct transplantable epithelia by tissue engineering using keratinocyte cell cultures enriched in stem cells which give rise to a self-renewing tissue. While the in vivo approaches turned out to be inefficient,

Correspondence: Dr U Griesenbach, Department of Gene Therapy, Emmanuel kaye Building, NHLI, Imperial College, Manresa Road, London, UK.

E-mail: u.griesenbach@imperial.ac.uk

Received 24 August 2007; accepted 24 August 2007 transduction of epidermal stem cells using oncoretroviral vectors succeeded in fully correcting the genetic defects in keratinocytes obtained from patients with X-linked ichthyosis, lamellar ichthyosis, xeroderma pigmentosum, junctional and dystrophic epidermolysis bullosa (JEB and DEB respectively) both in vitro and ex vivo. ${ }^{1}$

However, despite the encouraging results of the preclinical studies, evidence of clinical efficacy has been obtained so far only for JEB, a severe recessive skin blistering condition due to defects in genes for the keratinocyte adhesion molecule laminin $5 .^{2}$ The small size of the transgene required to cure the genetic defect in patients with an abnormal expression of the beta3 chain of laminin 5, and the restoration of the adhesion properties of the transduced JEB keratinocytes facilitated the implementation of a pilot phase-I clinical trial on a single adult patient. This pioneering work took advantage of the fact that:

(1) the recombinant retrovirus targeted almost $100 \%$ of the epithelial stem cells in vitro; 
(2) the selected patient expressed low levels of the mutant protein, which prevents an immune response to the transgene product;

(3) the grafted areas of the body had non-healing lesions due to the absence of epithelial stem cells able to compete with the transplanted epithelia in the healing process.

After nearly 2 years of follow-up the study has provided proof-of-principle for the feasibility and safety of a transduction/transplantation strategy that might be applicable to other genetic skin disorders. However, the successful treatment of JEB by gene therapy needs to be confirmed by clinical trials with a larger number of patients to establish statistical significance in terms of the definition of the clinical parameters required for inclusion of candidates, and the constellation of genetic mutations treatable without induction of immune responses. Due to the extreme rarity of JEB patients, a consortium of European groups is currently preparing a trans-national recruitment for clinical studies to be carried out in the next years. However, for approval of these trials, safety concerns for the potential genotoxicity of the retroviral vectors must be met, notably, the development of selfinactivating vectors safer than the first generation oncoretroviral backbone used in the pilot assay.

Despite these significant advances, the question remains as to whether a similar ex vivo approach can be extended to other genetic skin disorders with a medical and societal impact larger than JEB, including dominant inherited conditions. While the need for vectors presenting the best compromise in terms of capacity to accommodate large DNA fragments, transfer efficiency, safety, and biodistribution, undoubtedly remains a major challenge, the immune reaction in patients lacking expression of a gene product raises questions which at present have no answer.

\section{Huntington's disease}

\section{A-C Bachoud-Lévi',2,3 and M Peschanskit ${ }^{1}$ INSERM, U841, NPI, Creteil, France; '2ENS, DEC, Paris, France; ${ }^{3} A P-H P$, Hospital Henri-Mondor, Sce de Neurologic, Creteil, France; "INSERM/UEVE, U861, I-Stem, AFM, Evry, France}

Huntington's disease (HD) is a monogenic dominantnegative neurodegenerative disease caused by the expansion of CAG repeats in the IT5 gene on Chromosome 4 encoding huntingtin. HD occurs in adulthood (30-50 years of age) and is fatal within 15-20 years of diagnosis. Since identification of the HD's gene mutation, the use of gene therapy has been envisaged. Downregulation of the mutant IT5 allele while leaving the normal allele intact, is effective in in vitro models. Intrastriatal injection of interference RNA directed against mutant human huntingtin reduced protein expression and improved behavioural and pathological abnormalities in transgenic mice. ${ }^{3,4}$ However, numerous technical issues have to be solved before embarking on a human trial involving the delivery of RNAi to the brain.

Gene therapy using neurotrophic factors such as NGF, BDNF or CNTF (please define abbreviations) has also been postulated for the treatment of HD. CNTF deserves special attention. The protein reduced neuronal death of the striatal neurons in various experimental models mimicking HD and is, to date, the only gene therapy tested in patients. Adenoviral ${ }^{5}$ and lentiviral vectors ${ }^{6}$ were used to deliver CNTF into the striatum of rodent and primate HD models and showed neuronal survival and prevented behavioural deterioration. The only phase I gene therapy trial currently performed in HD patients used encapsulated genetically-engineered BHK cells over-expressing CNTF. ${ }^{7}$ BHK cells were placed into polymer capsules formed by a semi-permeable membrane and introduced, via stereotactic neurosurgery, into the right lateral ventricle of six HD patients. Capsule membranes contain pores, which allow the release of CNTF and the entry of oxygen and nutrients, but prevent the penetration of large proteins and host cells, and thus protects the cells from a host immune reaction. This strategy allows the use of xenogenic engineered cells and prevents proliferation of cells outside the capsule. The trial lasted 2 years with a replacement of capsules every 6 months. The procedure proved to be safe. However, only 11 out of the 24 retrieved capsules still released quantifiable amounts of CNTF after 6 months. Primate models had shown that implantation of 4 capsules was required to achieve correction of the phenotype. The clinical trial, however, was primarily a safety trial and only 1 capsule was implanted per patient. It was, therefore, not surprising that there was no clear clinical benefit in the patients. Nevertheless, surrogate marker (long latency reflex and somatosensory evoked potentials) improved in three out of six patients. A phase II trial allowing assessment of the therapeutic potential of CNTF gene therapy to the brain in HD patients is currently waiting for more efficient delivery vectors. Gene therapy is a source of hope for the cure or the improvement of HD but in vivo experimentation in animal models are still required to meet the challenge of brain gene transfer.

\section{Alzheimer's disease}

\section{A Blesch ${ }^{1}$ and MH Tuszynski, ${ }^{1,2,3}$ ${ }^{1}$ Department of Neurosciences, University of California, San Diego, La Jolla, CA; ${ }^{2}$ Veterans Administration Medical Center, San Diego, CA}

The nervous system growth factor Nerve Growth Factor (NGF) prevents the death of, and stimulates the function of, cholinergic neurons in the rodent and primate central nervous system. Further, NGF administration improves learning and memory in lesioned and aged rats, and improves neuronal function and prevents cell death in a mouse model of amyloid overexpression. ${ }^{8-10}$ These potent effects of NGF on cholinergic systems in the brain are of clear relevance to patterns of neuronal degeneration in the most common human neurodegenerative disorder, Alzheimer's disease (AD). However, to test the hypothesis that NGF will reduce neuronal degeneration and slow cognitive decline in $\mathrm{AD}$, a satisfactory method of NGF delivery to the brain is required.

In 2001 we initiated a trial of ex vivo NGF gene delivery in early stage AD. To restrict NGF delivery to the basal forebrain in a localized and targeted manner, genetically modified cells served as biological minipumps to provide therapeutic levels of NGF to basal 
forebrain neurons. Experiments in rodents and primates confirmed that grafts of NGF-expressing autologous fibroblasts were equally effective compared to intraventricular NGF protein infusions in preventing cholinergic neuronal degeneration and stimulating cell function. Additional dose escalation studies confirmed the safety and persistence of NGF gene expression over time periods of at least one year in the non-human primate brain. Based on the extensive pattern of efficacy and safety demonstrated after ex vivo NGF gene delivery, a Phase I trial of ex vivo NGF gene delivery was initiated in 8 subjects diagnosed with early AD. ${ }^{11}$ Fibroblasts obtained from skin biopsies were genetically modified using Moloney leukemia virus (MLV) retroviral vectors to express NGF, and these autologous cells were stereotactically implanted adjacent to the Nucleus basalis of Meynert (NBM) region of the brain. This brain region (of approximately $1 \mathrm{~cm}$ length and $3 \mathrm{~mm}$ width) contains degenerating cholinergic cell bodies in $\mathrm{AD}$, and can be practically targeted using techniques of ex vivo gene delivery, yet this compact nucleus extends axonal projections throughout the entire cortex, allowing NGF to influence diverse cortical regions by targeting of the much smaller Nucleus basalis.

Surgery for stereotaxic injections of cells was initially conducted in patients that were awake but sedated. However, two patients abruptly moved during the surgical session causing brain hemorrages. To mitigate this risk, all other subjects underwent surgery under deep sedation or general anesthesia, and no further surgical adverse effects occurred. With follow-up now available for up to 5 years, no adverse events related to either NGF or viral vector delivery have occurred. PET scans in bilaterally-treated subjects indicated a significant increase in metabolic activity throughout the cortex, consistent with potential widespread modulation of cortical activity by NGF effects on the Nucleus basalis. Cognitive testing suggested potential slowing of cognitive decline, although reliable conclusions in this respect cannot be drawn from a small sample of subjects lacking placebo controls or blinded assessments. In addition, histological analysis of the brain of one of the subjects, who died 5 weeks following gene delivery, demonstrated sprouting of cholinergic axons from the Nucleus basalis into NGF secreting grafts, a classic 'trophic effect' of the sort previously observed in rodent and primate studies. This anatomical response to NGF clearly established that degenerating cholinergic neurons in the $\mathrm{AD}$ brain are capable of responding to NGF.

As gene delivery technology advanced in the period following the initiation of the above Phase I trial, preclinical studies were repeated using AAV-NGF gene delivery. AAV-NGF injections in the brains of rodents and primates reproduced the full spectrum of biological efficacy observed in previous animal studies, with no evidence of nervous system toxicity. Based on these findings, a second Phase 1 trial of AAV-NGF gene delivery was recently conducted in early and mid-stage $\mathrm{AD}$, sponsored by Ceregene, Inc. ${ }^{12}$ As the phase I AAVNGF trial reaches a close, a multi-centre phase II sham surgery controlled, double-blinded trial in AD is planned. The phase II trial will explore the potential effect size of NGF gene delivery on cognitive decline in $\mathrm{AD}$, advancing a program that, if successful, will provide a potential opportunity to fundamentally alter the progressive and tragic course of this common human disorder.

Conflict of Interest Statement: $\mathrm{AB}$ and MHT have a financial interest in Ceregene, Inc.

\section{HIVIAIDS}

\section{DS Strayer}

Department of Pathology, Jefferson Medical College, Philadelphia, PA, USA

The challenge in HIV/AIDS gene delivery has been to devise strategies to transduce sufficient HIV-susceptible cells durably enough to provide adequate antiretroviral protection. Recent clinical gene transfer studies give reason for optimism that this goal is feasible. ${ }^{13,14}$

Transgenes. Many transgenes protect HIV-susceptible cells from HIV infection and/or replication in vitro. These include genes encoding proteins that inactivate or inhibit HIV-1 proteins, or that make the cellular environment less hospitable to HIV-1 infection or replication. Untranslated RNA-antisense, ribozymes, decoys and interfering RNA-similarly may target viral or cellular functions needed for HIV-1 infection and replication. Many transgenes confer potent anti-lentiviral protection. Their specific structures and functions are probably less critical than the means by which they are delivered, the cells to which they are delivered and how long they persist.

Therapeutic strategies. Clinical gene transfer studies for HIV/AIDS generally target hematopoietic stem cells (HSC) or peripheral blood T cells (therapeutic immunization is a separate topic.) Recent human studies involving HSC and T cell transduction have begun to offer hope as clinically feasible strategies.

(a) $T$ cells. Recent early phase clinical studies report that ex vivo gene delivery to stimulated blood $\mathrm{T}$ cells using lentiviral or retroviral vectors, followed by expansion and reinfusion, has so far been safe. ${ }^{13,14}$ In most patients percentages of circulating lymphocytes (PBL) carrying the transgene decline, often becoming undetectable by 6 months. However, gene-marked blood cells persist at low levels in some subjects. ${ }^{13,14}$ As most patients are simultaneously treated with highly active antiretroviral pharmacotherapy (HAART), the therapeutic effectiveness of transplanting gene-modified PBL is difficult to assess, but potentially promising data are reported. ${ }^{13}$ Since this approach targets mature circulating cells, rather than progenitors, it probably is safer than HSC gene delivery. However, as PBL have finite life expectancies, repeated transplants would probably be necessary to maintain therapeutic effect.

(b) HSC. Most early studies of gene transfer for HIV / AIDS involved transduction of HSC. ${ }^{15}$ This approach offers the potential advantage of transducing progenitors of several populations of HIV-susceptible cells. Ex vivo stimulation and transduction are needed because oncoretroviral and lentiviral vectors transduce resting HSC poorly. Transduced cells are then reimplanted. HSC gene transfer promises a permanent supply of HIV-resistant derivative cells of multiple lineages. Without pretransplant conditioning engraftment is inefficient, yet the most effective conditioning-bone marrow ablation-is impracticable as routine AIDS therapy. Results have been 
mixed: levels of gene-modified blood cells generally become low or undetectable within months of transplantation. There is, however, evidence that gene-modified, HIV-resistant mature cells persist and have a survival advantage $^{16}$ compared to their unmodified cousins. Reports of tumors after human HSC gene delivery with oncoretroviral vectors mandate proceeding cautiously.

Strategies to increase percentages of transgene-positive blood cells. Clinical T cell and HSC gene transfer trials suggest that for HIV/AIDS gene therapy to be clinically useful percentages of transduced cells must be increased. Thus, strategies employing in vivo selection are being considered. Gene transfer of methylguanine methyltransferase (MGMT) followed by nitrosourea treatment (BCNU) shows promise in selecting for gene-modified cells, especially as substrates less toxic than BCNU are identified. ${ }^{17}$ Other approaches to selection and HSC transduction used in human and animal studies have also received attention.

Conclusions and future prospects. Recent clinical experiments using gene transfer to treat HIV/AIDS have emphasized T cell-directed gene delivery, ex vivo expansion and reinfusion. Improvements in HSC-directed gene transfer also offer promise. These human studies suggest that progress is being made, in terms of both improved persistence of gene-modified cells and antiretroviral effect, and are cause for optimism.

\section{Ornithine transcarbamylase deficiency}

\section{G Gao and JM Wilson}

Gene Therapy Program, Department of Pathology and Laboratory Medicine, University of Pennsylvania, Philadelphia, PA

The liver is an important target for gene therapy because of the central role it plays in a variety of metabolic pathways and in the production of serum proteins. The first clinical trial of gene therapy for the liver was based on transplantation of ex vivo modified homologous hepatocytes in patients with familial hypercholesterolemia. The subsequent development of viral vectors capable of targeting cells in vivo suggested a more practical and efficient approach for genetically modifying the liver in which the vector is simply infused into the circulation gaining access to hepatocytes through the fenestrated endothelium.

The first demonstration of correction of a liver metabolic defect in an animal model following in vivo vector delivery used a recombinant adenovirus infused into newborn mice with a deficiency of ornithine transcarbamylase (OTC). ${ }^{18} \mathrm{~A}$ deficiency of this enzyme in humans leads to a syndrome of repeated episodes of life threatening hyperammonemia. The mouse models have a partial defect of OTC and a similar phenotype. We began to develop the adenovirus platform for evaluation in research subjects with OTC deficiency. Prior to a consideration in humans, we developed improved generations of the vector to attenuate further expression of viral genes and diminish the associated toxicity. A phase I study was initiated in which adults with a partial deficiency of OTC received a third generation adenoviral vector expressing OTC via infusion into the hepatic artery. The phase I dose escalation study progressed through 6 cohorts with evidence of modest gene transfer (by in situ hybridization of liver biopsies) and some toxicity. ${ }^{19}$ The second research subject in the highest projected dose developed a very different response to therapy than the previous 17 subjects that led to severe systemic inflammation and lethal multi-organ failure. ${ }^{20}$ Subsequent studies suggested this was caused by an exaggerated and unexplained innate immune response to the input vector capsids.

OTC deficiency remains an important model for the evaluation of novel liver-directed gene therapies. Children born with a complete deficiency of OTC suffer severe morbidity and premature mortality despite state-of-theart pharmacologic, dietary and supportive clinical treatments. The relative success of liver allograft transplants in this disease indicates that hepatocyte gene transfer should be curative. Promising new vectors based on adeno-associated viruses have shown tremendous promise in mouse models of OTC deficiency. ${ }^{21}$ This vector platform is capable of very efficient targeting of hepatocytes in vivo without apparent activation of innate immunity and long-term transgene expression. Transgene-specific T cell responses appear to be blunted in the context of AAV delivered to the liver, which will help reduce toxicity and improve efficacy when treating patients with deletions, frame shift or premature stop mutations in the OTC gene. One concern that requires additional investigation prior to embarking on clinical trials is the oncogenicity of the vector and the activation of $\mathrm{T}$ cells to the input AAV capsids.

\section{Hemophilia}

\section{KA High}

The Children's Hospital of Philadelphia, Philadelphia, $P A$, USA

Hemophilia is the $\mathrm{X}$-linked bleeding disorder caused by mutations in the genes encoding Factor IX (an enzyme) or Factor VIII (cofactor for the enzyme), both required for normal blood coagulation. Despite many advances, attempts to manage a lifelong disease by intravenous infusion of a protein with a relatively short half-life present major obstacles. Moreover, the cost of clotting factor concentrates (typically $\$ 50000-\$ 100000 /$ year for an adult with severe disease) has been prohibitive, in fact, out of reach, for the majority of the world's hemophilia population. These factors have fuelled interest in developing alternate therapeutic approaches such as gene transfer. At the outset, hemophilia was deemed an appealing target for gene transfer, not only because of the relatively large population of affected individuals, but also because the therapeutic gene does not need to be tightly regulated, and even modest increases in circulating levels of clotting factor (e.g. from $<1 \%$ to $\sim 5 \%$ ) result in a substantial improvement in symptoms, effectively converting severe disease to a mild phenotype.

Multiple strategies were pursued, in phase I/II trials involving a total of 41 patients with severe hemophilia A or B. These trials were sponsored by biotechnology companies including Transkaryotic Therapies (ex vivo transduction of autologous fibroblasts by a plasmid expressing a truncated Factor VIII molecule [B-domain deleted, BDD] $)^{22}$ Chiron Corporation (intravenous infusion of a retroviral vector expressing BDD F.VIII), ${ }^{23}$ Avigen, Inc. (AAV-mediated gene 
transfer into skeletal muscle [first trial] and liver [second trial] of a Factor IX minigene) ${ }^{24}$ and GenStar Corporation (intravenous infusion of a gutted adenoviral vector expressing Factor VIII).

All of these trials were first-in-class, i.e. the first instance of a particular vector being used in a particular target tissue, and thus yielded important safety data for the approaches under investigation. None, however, resulted in long-term expression of the clotting factor at therapeutic levels. Each approach encountered a critical issue, either in terms of safety or efficacy, that required further laboratory or clinical investigation. In the wake of these disappointing initial results, more rigorous analysis of pre-clinical data in the hemophilic dog model has supported continued pursuit of some strategies and modification or abandoning of others. Circulating factor levels in the range of $5-25 \%$ have now been achieved in hemophilic dogs or non-human primates using three distinct approaches: a retroviral vector can be infused into neonatal dogs, where hepatocytes are still rapidly dividing, and yield high-level clotting factor expression; ${ }^{25}$ AAV vectors can be delivered to skeletal muscle via an intravascular route and can transduce a large number of muscle fibers yielding therapeutic levels; ${ }^{26}$ or AAV vector can be delivered to liver via the hepatic artery, the portal vein, or in the case of AAV-8 vectors, intravenously, to yield therapeutic levels of clotting factors. ${ }^{27}$

The retroviral approach will require either very young subjects (in utero or neonatal, where hepatocytes are rapidly dividing), or some pharmacologic or surgical intervention to induce hepatocyte replication in older subjects. This approach is not actively being pursued for hemophilia. AAV transduction of skeletal muscle is being actively investigated in clinical trials for the muscular dystrophies, and pursuit of this approach for hemophilia will likely depend on development of intravascular (rather than intramuscular) delivery techniques that are likely to be pursued first in a setting of muscle disorders. Delivery of AAV vector to the liver for hemophilia has shown the most promising results to date, having achieved circulating levels of $\sim 10-12 \%$ in one clinical study, although the duration of expression was only weeks, as opposed to years in hemophilic dogs. ${ }^{24}$ The etiology of the shortened duration of expression that occurred in humans but not in animal models appears to be the presence of memory $\mathrm{CD}^{+} \mathrm{T}$ cells to AAV capsid, found in humans because they are the only natural hosts for wild-type AAV-2 infection. Two trials of AAV in liver, reflecting two possible solutions to this dilemma (transient immunosuppression to block the immune response to capsid until it is cleared from the cell or the use of an alternate AAV serotype [AAV-8] to which humans are not naturally exposed) have been proposed and should be investigated within the next year.

\section{ADA-SCID}

\section{A Aiuti' ${ }^{1}$ F Cattaneo ${ }^{1}$ and MG Roncarolo ${ }^{1,2}$ ${ }^{1}$ Pediatric Clinical Research Unit, San Raffaele Telethon Institute for Gene Therapy (HSR-TIGET); '2Vita-Salute San Raffacle Univesity, Milano, Italy}

Severe Combined Immunodeficiency (SCID) due to the lack of adenosine deaminase (ADA) is a rare, fatal, disorder characterised by immunological defects and organ/systemic toxicity caused by the accumulation of purine metabolites. In the absence of an HLA-identical sibling donor, hematopoietic stem cell (HSC) transplantation from alternative donors is restricted by high morbidity and mortality, while enzyme replacement therapy (PEG-ADA) often fails to sustain long-term immunity. Since September 1990, over 35 ADA-SCID patients have been enrolled in phase I/II gene therapy trials in 6 different centres worldwide. Gene therapy was based on infusions of autologous peripheral blood lymphocytes (PBL) or HSC transduced ex vivo with retroviral vectors encoding ADA.

Long-term follow up of children receiving PBL gene therapy has demonstrated long-term persistence of gene corrected $\mathrm{T}$ cells $>12$ years after the last infusion, without adverse events. ${ }^{28}$ However, all patients were maintained on PEG-ADA therapy, abrogating the potential selective growth advantage for gene corrected cells and preventing the full evaluation of gene therapy efficacy. Discontinuation of PEG-ADA in one patient treated with engineered PBL led to in vivo selection for transduced $\mathrm{T}$ cells with improvement of immune functions, but not complete metabolic detoxification. ${ }^{29}$

The cumulative experience from pilot HSC gene therapy studies showed an inadequate level of engraftment of transduced cells, supporting the need for patients' pre-conditioning in order to achieve long-term engraftment of multipotent HSC and full correction of ADA-SCID phenotype. For this purpose, a gene therapy protocol, initiated in 2000, combined infusion of retrovirally transduced bone marrow $\mathrm{CD}_{3} 4^{+}$cells with lowdose busulfan conditioning. ${ }^{30}$ In the first two patients reported, long-term engraftment of multilineage HSC, differentiating in both myeloid and lymphoid transduced cells, resulted in sustained ADA activity and efficient systemic detoxification. Gene therapy led to an improvement of cellular and humoral responses in the absence of enzyme replacement therapy, with proven clinical benefit. ${ }^{30}$ The initial results have now been extended in a larger cohort of patients (at present 12 patients have been treated), and none of them showed adverse events related to gene transfer.

Engraftment of ADA-carrying cells and sustained biochemical and immunological correction was recently obtained in a patient treated in the UK using melphalan as a pre-conditioning drug. ${ }^{31}$ An important limitation for the use of autologous transduced HSC may be represented by the low number of stem cells available ${ }^{30}$ in the bone marrow or by pre-existing chromosomal alterations $^{32}$ in progenitor cells. Another critical issue is the potential risk of insertional mutagenesis related to the use of retroviral vectors. Our analyses of a large collection of integration sites in ADA-SCID patients treated with gene therapy showed no clonal expansion and the absence of in vivo skewing of the integration profile. ${ }^{33}$

In summary, results of the latest clinical trials have provided evidence for safety and efficacy of gene therapy for ADA-SCID, establishing as a paradigm for other genetic diseases, the use of reduced intensity conditioning in facilitating the engraftment of gene corrected HSC. An extended follow up of gene therapy treated patients will be required to confirm these results and to compare the outcome of gene therapy with other treatment, in order to obtain its recognition as an approved therapy. 


\section{Cancer: immunotherapy}

\section{AM Leen and MK Brenner \\ Center for Cell and Gene Therapy, Baylor College \\ of Medicine, Texas Children's Hospital, and The \\ Methodist Hospital, Houston, Texas}

Despite the success of 'passive' immunotherapy with monoclonal antibodies, treatment of cancer by active immunization or by adoptive transfer of a cellular immune response has proven problematic. Most tumors express weak self-antigens to which the host is tolerant, and they lack the co-stimulatory molecules necessary for full recruitment of cellular immunity. Many tumors also employ active immune evasion strategies. ${ }^{34}$ Gene transfer is being used to overcome these obstacles and the combination of cellular and gene therapy is now producing consistent rather than anecdotal success in an increasing number of tumors.

Increasing antigenic stimulation from tumor cells and their microenvironment: Gene transfer can be used to increase the 'visibility' of tumors to the immune system by in vivo or ex vivo modification of malignant cells, using genes encoding immunostimulatory cytokines (e.g. TNF- $\alpha$, IFN- $\alpha$ ), or co-stimulatory molecules (e.g. CD40 ligand). Both approaches augment in vivo tumor antigen presentation and help to recruit tumor-specific T cells and B cells. Alternatively, professional Antigen Presenting Cells (APCs) can be modified using tumor-peptides, recombinant protein, tumor lysates, RNA, DNA, or viral vectors, to promote the activation of effector $\mathrm{T}$ cells in vivo. ${ }^{35}$ Recently the FDA recommended approval for the first genetically modified Dendritic Cell (DC) vaccine (for recurrent prostate cancer), opening the door for future new agents of this type. Meanwhile current early phase clinical studies are optimizing the immunostimulatory efficacy of DC vaccines, by augmenting expression of costimulatory molecules (such as CD40 ligand), enhancing their secretion of immunostimulatory cytokines, or by increasing their lifespan ${ }^{36}$.

Modification of effector T cells: Sustained tumor elimination usually requires the presence and persistence of large numbers of effector T cells. While active vaccination attempts to recruit these cells in vivo, the inhibitory environment produced by cancer cells impedes this process. An alternative is to prepare effector $\mathrm{T}$ cells ex vivo and adoptively transfer them to the patient. Tumor specificity can be induced through the selection of subpopulations expressing the appropriate native $\mathrm{T}$ cell receptor, or by transfer and expression of tumor specific receptors derived from antibodies or from the $\alpha \beta$ TCR of tumor specific $\mathrm{T}$ cell clones. For example, Morgan and colleagues expressed the $\alpha$ and $\beta$ chains of an antiMART-1 TCR in T cells from 15 patients with melanoma, 2 of whom had complete tumor regression. ${ }^{37}$

We can improve the functionality as well as the specificity of adoptively transferred $\mathrm{T}$ cells. We can increase homing to tumor sites by expressing chemokine receptors specific for the molecules the tumors produce, while $\mathrm{T}$ cell expansion and persistence in the hostile tumor microenvironment can be increased by expressing transgenes that confer resistance to apoptosis, senescence, or inhibitory cytokines, or that provide autocrine T cell growth. ${ }^{38}$ Any genetic strategy that enhances the survival of $\mathrm{T}$ cells, however, runs the risk of producing unwanted lymphoproliferation. Although protocols transferring genes to $T$ cells have been safe to date, this strategy is being implemented with caution. Finally, the in vivo expansion and persistence of adoptively transferred immune cells can be increased by prior lymphodepletion of the patient with drugs, radiation or monoclonal antibodies, thereby removing unwanted inhibitory $\mathrm{T}$ regulatory cells, and favoring homeostatic expansion of the infused anti-tumor T cells.

\section{Cancer: oncolytic viruses}

\section{IA McNeish and NR Lemoine Centre for Molecular Oncology, Barts and the London Queen Mary's School of Medicine and Dentistry}

Oncolytic viruses multiply selectively within cancers, causing cell death, with released mature viral particles infecting neighbouring cells. The tumour suppressor and cell defence mechanisms that viruses subvert are the same as those lost in carcinogenesis. Some viruses are intrinsically tumour-selective in their replication, whilst the large DNA viruses require deletion of key viral genes to achieve selectivity.

Although anecdotal reports of virus-induced antitumour responses appeared over 100 years ago, the first formal clinical trials tested the E1B-55K deleted adenovirus dl1520 (Onyx-015), which was hypothesised to replicate selectively in p53-negative cells. At least 15 clinical trials using $d l 1520$ have been completed and a derivative, H101, is licensed in China. However, single agent activity was poor, with minimal systemic efficacy and the mechanism of activity was unrelated to p53 status: in addition to inhibition of p53, E1B 55K promotes the export of late viral mRNA from the nucleus. Tumour cells have altered mechanisms for export of these RNA species and thus do not rely on E1B 55K. Nonetheless, doses of up to $10^{13}$ particles were safely administered by a variety of routes. Clinical trials of second generation adenoviral mutants, such as dl922-947 or $\Delta 24$, have not yet commenced. Three clinical trials of transcriptionallyregulated adenoviruses, with the Prostate-specific Antigen (PSA) promoter/enhancer elements driving E1 expression, have been completed in hormone-refractory prostate cancer. CV706 and CG7870 were administered intratumourally and intravenously respectively with no significant toxicities and some transient PSA responses. ${ }^{39}$

All Herpes simplex viruses tested in clinical trials to date are deleted in one or both copies of the ICP34.5 gene, which inhibits the interferon/protein kinase $\mathrm{R}$ response to viral infection. 1716 has been safely given by direct injection in melanoma and glioma at doses of $10^{5}$ pfu, whilst G207, which is also deleted in ICP6 (ribonucleotide reductase), has been given by intratumoural injection at doses of up to $3 \times 10^{9} \mathrm{pfu}$ in glioma with no dose limiting toxicities. OncoVex ${ }^{\mathrm{GM}-\mathrm{CSF}}$ is based upon a clinical HSV1 isolate, rather than laboratory strains, and is deleted in both ICP34.5 and ICP47 (to increase MHC class I presentation) and encodes GM-CSF. Doses of up to $10^{8} \mathrm{pfu} \mathrm{ml}^{-1}$ were given by direct intratumoural injection to cutaneous metastases or melanoma, with evidence of local, but not distant, responses. ${ }^{40}$

A tk-negative Vaccinia virus mutant encoding GMCSF was tested in preliminary clinical trials in 1999, with 
evidence of local responses in melanoma metastases injected with up to $2 \times 10^{7}$ pfu twice weekly. ${ }^{41}$ There is also now renewed interest in Vaccinia, but no trial has yet been completed or published.

Of the naturally occurring oncolytic viruses, Newcastle Disease Virus (NDV) and Reovirus have been studied in phase I/II trials. NDV is a single-stranded paramyxovirus that, like ICP34.5 ${ }^{-}$HSV mutants, exploits defects in the interferon response pathway to achieve tumour selectivity. In initial studies, intravenous doses of $1.2 \times 10^{11} \mathrm{pfu} \mathrm{m}^{-2}$ were associated with dose-limiting diarrhoea and flu-like symptoms. However, using an intra-patient dose-escalation desensitisation regime, the same doses could be administered with reduced toxicity. ${ }^{42}$ In the two trials, objective responses were seen in six patients. Reovirus, a double stranded RNA virus, replicates in cells with deregulated Ras signalling. Direct intra-tumour injections of up to $10^{9} \mathrm{pfu}$ were given in glioma safely, but without objective responses. ${ }^{43}$ Phase II studies in conjunction with radiotherapy in patients with cutaneous metastases are on-going (K Harrington, Personal communication).

In summary, several oncolytic viruses have been tested in clinical trials, with impressive safety records. All can induce anti-tumour responses after direct injection, but systemic activity remains a challenge. Strategies for the immediate future will include arming viruses with transgenes and combining with chemo- and/or radiotherapy. For example, a phase II study of OncoVex GM-CSF with chemoradiotherapy in locally advanced head and neck cancers is on-going. However, in the longer term, gaining a fuller understanding of host cell factors that determine viral sensitivity as well as manipulating the immune responses to both virus and cancer cell will be essential if these agents are to become standard therapies for cancer.

\section{Cancer: suicide gene therapy}

\section{EA Chiocca ${ }^{1}$ and DJ Waxman ${ }^{2}$}

${ }^{1}$ The Ohio State University Medical Center, Columbus, $\mathrm{OH}$; ${ }^{2}$ Boston University, Boston, $\mathrm{MA}$

Suicide gene therapy, also known as prodrug-activation or gene-directed enzyme prodrug therapy, is a widely studied cancer gene therapy strategy, with numerous publications providing evidence of significant effects in animal models of cancer. $^{44}$ Translation into human clinical trials has included herpes simplex thymidine kinase (Hstk) gene transfer to induce susceptibility to acyclovir, ganciclovir or valacyclovir, bacterial cytosine deaminase (cd) gene transfer to induce susceptibility to 5-fluorocytosine, and mammalian cytochrome P450 2B (CYP2B) gene transfer ${ }^{45}$ to enhance chemosensitivity to cyclophosphamide and ifosfamide, among others.

An early phase III clinical trial of ganciclovir/Hstk gene therapy for patients with malignant glial brain tumors failed to show significant effects, ${ }^{46}$ which may in part reflect low transduction efficiency of the retroviral producer cells employed. More encouraging results are emerging from the use of adenoviral vectors (either replication-defective or oncolytic) in phase I and phase II trials where Hstk or an Hstk/cd combined transgene was employed for prostate ${ }^{47}$ and other cancers. Some studies have suggested that adenoviral vectors enhance biodis- tribution of the transferred gene compared to retroviral vectors, while others have suggested that the immune response associated with expression of a suicide gene is an important contributor to the anticancer response. The efficacy of intratumoral administration of a replicationdefective adenovirus bearing Hstk may soon be known from results of a large phase III clinical trial for human malignant glioma about to be completed in Europe.

Refinements will undoubtedly be required. Oncolytic viruses delivering suicide genes may help address issues relating to the inefficiency of vector biodistribution and suicide gene delivery. Determination of whether the observed inflammatory response, particularly in the initial phases, is beneficial by setting up an adaptive immune response, blank or is detrimental by its elimination of vector and transgene is also of importance. Promising avenues of research include the identification of combinations of therapies that synergize with suicide gene therapy (e.g. radiation, chemotherapy) or combinations based on new prodrug-activation paradigms. Imaging analysis of gene transfer may provide useful correlates of gene therapy responses in humans. Finally, tumor distribution of prodrug and the use of mutants or species variants of the suicide gene that bioactivate the prodrug with higher catalytic efficiency also need to be explored. Ultimately, the future of suicide gene therapy for cancer will depend on a successful phase III trial. The road to such a trial is expensive and fraught with potential roadblocks. However, the plight of patients with advanced cancers requires that such a journey be undertaken through carefully designed studies that maximize the scientific knowledge gained and help establish which cancers, and which patients, are likely to most benefit from this therapeutic strategy.

\section{References}

1 Del Rio M, Gache Y, Jorcano JL, Meneguzzi G, Larcher F. Current approaches and perspectives in human keratinocyte-based gene therapies. Gene Ther 2004; 11 (Suppl 1): S57-S63.

2 Mavilio F, Pellegrini G, Ferrari S, Di Nunzio F, Di Iorio E, Recchia A et al. Correction of junctional epidermolysis bullosa by transplantation of genetically modified epidermal stem cells. Nat Med 2006; 12: 1397-1402.

3 Harper SQ, Staber PD, He X, Eliason SL, Martins IH, Mao Q et al. RNA interference improves motor and neuropathological abnormalities in a Huntington's disease mouse model. Proc Natl Acad Sci USA 2005; 102: 5820-5825.

4 Rodriguez-Lebron E, Denovan-Wright EM, Nash K, Lewin AS, Mandel RJ. Intrastriatal rAAV-mediated delivery of anti-huntingtin shRNAs induces partial reversal of disease progression in R6/1 Huntington's disease transgenic mice. Mol Ther 2005; 12: 618-633.

5 Mittoux V, Ouary S, Monville C, Lisovoski F, Poyot T, Conde F et al. Corticostriatopallidal neuroprotection by adenovirusmediated ciliary neurotrophic factor gene transfer in a rat model of progressive striatal degeneration. J Neurosci 2002; 22: 4478-4486.

6 Zala D, Bensadoun JC, Pereira de Almeida L, Leavitt BR, Gutekunst CA, Aebischer P et al. Long-term lentiviral-mediated expression of ciliary neurotrophic factor in the striatum of Huntington's disease transgenic mice. Experimental Neurology 2004; 185: 26-35.

7 Bloch J, Bachoud-Lévi AC, Déglon N, Lefaucheur JP, Winkel L, Palfi $S$ et al. Neuroprotective gene therapy for Huntington's 
disease using polymer encapsulated cells engineered to secrete human CNTF: results of a phase I study. Human Gene Therapy 2004; 15: 968-975.

8 Hefti F. Nerve growth factor promotes survival of septal cholinergic neurons after fimbrial transections. J Neurosci 1986; 6: 2155-2162.

9 Fischer W, Wictorin K, Bjorklund A, Williams LR, Varon S, Gage FH. Amelioration of cholinergic neuron atrophy and spatial memory impairment in aged rats by nerve growth factor. Nature 1987; 329: 65-68.

10 Conner JM, Darracq MA, Roberts J, Tuszynski MH. Non-tropic actions of neurotrophins: subcortical NGF gene delivery reverses age-related degeneration of primate cortical cholinergic innervation. Proc Natl Acad Sci USA 2001; 98: 1941-1946.

11 Tuszynski MH, Thal L, Pay M, Salmon DP, U HS, Bakay R et al. A phase 1 clinical trial of nerve growth factor gene therapy for Alzheimer disease. Nat Med 2005; 11: 551-555.

12 Arvanitakis Z, Tuszynski MH, Bakay R, Arends D, Potkin S, Bartus $\mathrm{R}$ et al. Interim data from a phase 1 clinical trial of AAVNGF (CERE-110) gene delivery in Alzheimer's disease. American Acad Neurol 2007: Abstract online p05.071.

13 Levine BL, Humeau LM, Boyer J, MacGregor R-R, Rebello T, $\mathrm{Lu} \mathrm{X}$ et al. Gene transfer in humans using a conditionally replicating lentiviral vector. PNAS 2006; 103: 17372-17377.

14 Macpherson JL, Boyd MP, Arndt AJ, Todd AV, Fanning GC, Ely JA et al. Long-term survival and concomitant gene expression of ribozyme-transduced CD4+ T lymphocytes in HIV-infected patients. J Gene Med 2005; 7: 552-564.

15 Kohn DB, Bauer G, Rice CR, Rothschild JC, Carbonaro DA, Valdez $\mathrm{P}$ et al. A clinical trial of retroviral-mediated transfer of a Rev-responsive element decoy gene into CD34 cells from the bone marrow of human immunodeficiency virus-1-infected children. Blood 1999; 94: 279-287.

16 Podsakoff GM, Engel BC, Barbonaro DA, Choi C, Smogorzewska EM, Bauer $\mathrm{G}$ et al. Selective survival of peripheral blood lymphocytes in children with HIV-1 following delivery of an anti-HIV gene to bone marrow CD34+ cells. Mol Therapy 2005; 12: $77-86$

17 Brock CS, Newlands ES, Wedge SR, Bower M, Evans H, Colquhoun I et al. Phase I trial of temozolomide using an extended continuous oral schedule. Cancer Res 1998; 58: 4363-4367.

18 Stratford-Perricaudet LD, Levrero M, Chasse JF, Perricaudet M, Briand P. Evaluation of the transfer and expression in mice of an enzyme-encoding gene using a human adenovirus vector. Hum Gene Ther 1990; 1: 241-256.

19 Raper SE, Yudkoff M, Chirmule N, Gao GP, Nunes F, Haskal ZJ et al. A pilot study of in vivo liver-directed gene transfer with an adenoviral vector in partial ornithine transcarbamylase deficiency. Hum Gene Ther 2002; 13: 163-175.

20 Raper SE, Chirmule N, Lee FS, Wivel NA, Bagg A, Gao GP et al. Fatal systemic inflammatory response syndrome in a ornithine transcarbamylase deficient patient following adenoviral gene transfer. Mol Genet Metab 2003; 80: 148-158.

21 Moscioni $\mathrm{D}$ et al. Long-term correction of ammonia metabolism and prolonged survival in ornithine transcarbamylase-deficient mice following liver-directed treatment with adeno-associated viral vectors. Mol Ther 2006; 14: 25-33.

22 Roth DA, Tawa Jr NE, O'Brien JM, Treco DA, Selden RF. Nonviral transfer of the gene encoding coagulation factor VIII in patients with severe hemophilia A. N Engl J Med 2001; 344: 1735-1742.

23 Powell JS, Ragni MV, White II GC, Lusher JM, Hillman-Wiseman C, Moon TE et al. Phase 1 trial of FVIII gene transfer for severe hemophilia A using a retroviral construct administered by peripheral intravenous infusion. Blood 2003; 102: 2038-2045.

24 Manno CS, Pierce GF, Arruda VR, Glader B, Ragni M, Rasko JJ et al. Successful transduction of liver in hemophilia by AAVFactor IX and limitations imposed by the host immune response. Nat Med 2006; 12: 342-347.
$25 \mathrm{Xu} \mathrm{L}$, Gao C, Sands MS, Cai SR, Nichols TC, Bellinger DA et al. Neonatal or hepatocyte growth factor-potentiated adult gene therapy with a retroviral vector results in therapeutic levels of canine factor IX for hemophilia B. Blood 2003; 101: 3924-3932.

26 Arruda VR, Stedman HH, Nichols TC, Haskins ME, Nicholson M, Herzog RW et al. Regional intravascular delivery of AAV-2-F.IX to skeletal muscle achieves long-term correction of hemophilia B in a large animal model. Blood 2005; 105: 3458-3464.

27 Nathwani AC, Gray JT, McIntosh J, Ng CY, Zhou J, Spence Y et al. Safe and efficient transduction of the liver after peripheral vein infusion of self-complementary AAV vectors results in stable therapeutic expression of human FIX in nonhuman primates. Blood 2007; 109: 1414-1421.

28 Muul LM, Tuschong LM, Soenen SL, Jagadeesh GJ, Ramsey WJ, Long $\mathrm{Z}$ et al. Persistence and expression of the adenosine deaminase gene for 12 years and immune reaction to gene transfer components: long-term results of the first clinical gene therapy trial. Blood 2003; 101: 2563-2569.

29 Aiuti A, Vai S, Mortellaro A, Casorati G, Ficara F, Andolfi G et al. Immune reconstitution in ADA-SCID after PBL gene therapy and discontinuation of enzyme replacement. Nat Med 2002; 8: $423-425$.

30 Aiuti A, Slavin S, Aker M, Ficara F, Deola S, Mortellaro A et al. Correction of ADA-SCID by stem cell gene therapy combined with nonmyeloablative conditioning. Science 2002; 296: 2410-2413.

31 Gaspar HB, Bjorkegren E, Parsley K, Gilmour KC, King D, Sinclair $\mathrm{J}$ et al. Successful reconstitution of immunity in ADASCID by stem cell gene therapy following cessation of PEG-ADA and use of mild preconditioning. Mol Ther 2006; 14: 505-513.

32 Engel BC, Podsakoff GM, Ireland JL, Smogorzewska EM, Carbonaro DA, Wilson $\mathrm{K}$ et al. Prolonged pancytopenia in a gene therapy patient with ADA-deficient SCID and trisomy 8 mosaicism: a case report. Blood 2007; 109: 503-506.

33 Aiuti A, Cassani B, Andolfi G, Mirolo M, Biasco L, Recchia A et al. Multilineage hematopoietic reconstitution without clonal selection in ADA-SCID patients treated with stem cell gene therapy. J Clin Invest 2007; 117: 2233-2240.

34 Gattinoni L, Powell Jr DJ, Rosenberg SA, Restifo NP. Adoptive immunotherapy for cancer: building on success. Nat Rev Immunol 2006; 6: 383-393.

35 Nestle FO, Farkas A, Conrad C. Dendritic-cell-based therapeutic vaccination against cancer. Curr Opin Immunol 2005; 17: 163-169.

36 Hanks BA, Jiang J, Singh RA, Song W, Barry M, Huls MH et al. Re-engineered CD40 receptor enables potent pharmacological activation of dendritic-cell cancer vaccines in vivo. Nat Med 2005; 11: 130-137.

37 Morgan RA, Dudley ME, Wunderlich JR, Hughes MS, Yang JC, Sherry RM et al. Cancer regression in patients after transfer of genetically engineered lymphocytes. Science 2006; 314: 126-129.

38 Hsu C, Hughes MS, Zheng Z, Bray RB, Rosenberg SA, Morgan RA. Primary human $\mathrm{T}$ lymphocytes engineered with a codon-optimized IL-15 gene resist cytokine withdrawal-induced apoptosis and persist long-term in the absence of exogenous cytokine. J Immunol 2005; 175: 7226-7234.

39 Small EJ, Carducci MA, Burke JM, Rodriguez R, Fong L, van Ummersen $\mathrm{L}$ et al. A phase I trial of intravenous CG7870, a replication-selective, prostate-specific antigen-targeted oncolytic adenovirus, for the treatment of hormone-refractory, metastatic prostate cancer. Mol Ther 2006; 14: 107-117.

$40 \mathrm{Hu}$ JC, Coffin RS, Davis CJ, Graham NJ, Groves N, Guest PJ et al. A phase I study of oncoVEXGM-CSF, a second-generation oncolytic herpes simplex virus expressing granulocyte macrophage colony-stimulating factor. Clin Cancer Res 2006; 12: 6737-6747.

41 Mastrangelo MJ, Maguire Jr HC, Eisenlohr LC, Laughlin CE, Monken CE, McCue PA et al. Intratumoral recombinant GMCSF-encoding virus as gene therapy in patients with cutaneous melanoma. Cancer Gene Ther 1999; 6: 409-422. 
42 Hotte SJ, Lorence RM, Hirte HW, Polawski SR, Bamat MK, $\mathrm{O}^{\prime}$ Neil JD et al. An optimized clinical regimen for the oncolytic virus PV701. Clin Cancer Res 2007; 13: 977-985.

43 Forsyth P, Roldan G, George D, Wallace C, Morris D, Cairncross $\mathrm{J}$ et al. A phase I trial of intratumoral (i.t.) administration of reovirus in patients with histologically confirmed recurrent malignant gliomas (MGs). ASCO 2006, pp 1563.

44 Niculescu-Duvaz I, Springer CJ. Introduction to the background, principles, and state of the art in suicide gene therapy. Mol Biotechnol 2005; 30: 71-88.
45 Braybrooke JP, Slade A, Deplanque G, Harrop R, Madhusudan S, Forster MD et al. Phase I study of MetXia-P450 gene therapy and oral cyclophosphamide for patients with advanced breast cancer or melanoma. Clin Cancer Res 2005; 11: 1512-1520.

46 Rainov NG. A phase III clinical evaluation of herpes simplex virus type 1 thymidine kinase and ganciclovir gene therapy as an adjuvant to surgical resection and radiation in adults with previously untreated glioblastoma multiforme. Hum Gene Ther 2000; 11: 2389-2401.

47 Freytag SO, Stricker H, Movsas B, Kim JH. Prostate cancer gene therapy clinical trials. Mol Ther 2007; 15: 1042-1052. 